\title{
Stratégie canadienne de vaccination en cas de pandémie
}

\author{
B Henry ${ }^{1}$, S Gadient ${ }^{2}$ au nom du Groupe de travail sur la préparation du Canada en cas de grippe \\ pandémique ${ }^{3}$
}

\section{Résumé}

Le mandat de l'Agence de la santé publique du Canada consiste à se préparer et à réagir aux incidents de santé publique, notamment les pandémies de grippe. La préparation en cas de pandémie nécessite une approche à multiples facettes dans le cadre de laquelle tous les ordres de gouvernement collaborent. Le guide Préparation du Canada en cas de grippe pandémique : Guide de planification pour le secteur de la santé est un document d'orientation qui présente les activités de préparation clés du secteur de la santé conçues pour garantir que le Canada est prêt à réagir à la prochaine pandémie de grippe.

Le présent article, le premier d'une série, esquisse la stratégie canadienne de vaccination en cas de pandémie telle qu'elle est décrite dans l'annexe du document Préparation du Canada en cas de grippe pandémique portant sur les vaccins. La stratégie englobe l'ensemble des éléments constitutifs d'un programme de vaccination, notamment l'établissement de priorités pour la distribution initiale de vaccins, la sécurisation de l'approvisionnement en vaccins antipandémiques, l'innocuité des vaccins, la distribution et l'entreposage des vaccins, l'attribution et le niveau d'utilisation des vaccins.

Citation proposée : Henry B, Gadient S au nom du Groupe de travail sur la préparation du Canada en cas de grippe pandémique. Stratégie canadienne de vaccination en cas de pandémie. Relevé des maladies transmissibles au Canada. 2017 ;43(7/8):185-9. https://doi.org/10.14745/ccdr.v43i78a05f

\author{
Affiliations \\ ${ }^{1}$ Président du Groupe de travail \\ sur la préparation du Canada \\ en cas de grippe pandémique, \\ bureau de l'administrateur \\ provincial de la santé, Vancouver \\ (Colombie-Britannique) \\ ${ }^{2}$ Centre de l'immunisation \\ et des maladies respiratoires \\ infectieuses, Agence de la santé \\ publique du Canada, Ottawa \\ (Ontario) \\ ${ }^{3}$ Voir la section \\ "Remerciements " pour obtenir \\ une liste complète des membres \\ du groupe de travail
}

\section{*Correspondance : CPIPTGSecretariat- GTPCPSecretariat@phac-aspc. gc.ca}

\section{Introduction}

Les pandémies grippales sont des événements récurrents, bien qu'imprévisibles, qui surviennent lorsqu'un nouveau virus grippal A se répand largement et provoque un incident mondial. La planification liée à une urgence sanitaire à grande échelle et de longue durée dont les répercussions sont imprévisibles est difficile, mais essentielle. L'Agence de la santé publique du Canada (Agence) a pour mandat de se préparer et de réagir aux incidents de santé publique grâce à son leadership, aux partenariats et à la prise de mesures. Lors d'une pandémie grippale ou d'autres pandémies, l'Agence dirige la préparation et l'intervention du secteur de la santé à l'échelle nationale. Elle collabore avec des partenaires internationaux et représente le Canada au sein d'initiatives de sécurité sanitaire mondiales.

La préparation en cas de pandémie nécessite une approche à multiples facettes dans le cadre de laquelle tous les ordres de gouvernement (local, provincial/territorial, fédéral) collaborent. Parmi les composantes clés de la préparation du secteur de la santé figurent : la surveillance des maladies; les analyses en laboratoire et la recherche; le service de soins de santé et l'orientation en matière de santé publique; les communications; et les contre-mesures médicales visant à atténuer la transmission et à réduire le fardeau de la maladie grâce à l'utilisation d'antiviraux et de vaccins.

La pandémie de grippe H1N1 (pH1N1) de 2009 a été le tout premier test de la planification de la préparation du Canada en cas de pandémie. Elle a débouché sur une collaboration inédite entre les ordres de gouvernement et sur une implication réussie des intervenants. II s'est avéré très utile de tirer profit des réseaux de laboratoires existants tels que le Réseau des laboratoires de santé publique du Canada, et des systèmes de surveillance tels que le système national de surveillance de l'activité grippale au Canada, Surveillance de l'influenza.

Les planificateurs des services de santé ont beaucoup appris de la pandémie de grippe pH1N1 de 2009 (1). Mentionnons deux leçons importantes qui ont éclairé les considérations ultérieures relatives à la planification, lesquelles consistaient à veiller à ce que :

- les éléments déclencheurs de l'activation et de la dissolution d'aspects d'intervention précis soient bien définis;

- les plans d'intervention en cas de pandémie permettent une certaine adaptabilité et souplesse.

Cela témoigne du fait que l'incidence et la chronologie de l'activité pandémique du virus peuvent varier selon la région. Il se peut que chaque échelon de gouvernement (provincial, territorial et fédéral) ait ses propres facteurs à prendre en considération. Enfin, il est nécessaire de bien structurer les rôles et responsabilités des gouvernements fédéral, provinciaux et territoriaux en cas d'urgence de santé publique. 


\section{Planification nationale en cas de pandémie pour le secteur de la santé}

Le guide Préparation du Canada en cas de grippe pandémique : Guide de planification pour le secteur de la santé (PCGP) est un document d'orientation fédérale-provinciale-territoriale qui présente la manière suivant laquelle les territoires de compétence peuvent collaborer afin de garantir une approche coordonnée et uniforme du secteur de la santé en matière de préparation en cas de pandémie (2). Le corps du PCGP expose les principes fondamentaux, les concepts et les objectifs partagés qui sont indispensables à une préparation et une intervention efficaces en cas de pandémie. Les annexes reprennent ces éléments en fournissant les plans opérationnels techniques se rapportant à des enjeux précis tels que la surveillance, les mesures de santé publique et la stratégie de vaccination.

Le guide Préparation du Canada en cas de grippe pandémique est un document évolutif qui est régulièrement réexaminé et mis à jour à mesure qu'apparaissent de nouveaux renseignements et de nouvelles pratiques exemplaires. Le présent article est le premier d'une suite d'articles fournissant des mises à jour sur les différentes parties du guide Préparation du Canada en cas de grippe pandémique en résumant la Stratégie canadienne de vaccination en cas de grippe pandémique telle qu'elle est présentée dans l'Annexe sur les vaccins du guide Préparation du Canada en cas de grippe pandémique (3).

\section{Stratégie canadienne de vaccination en cas de pandémie}

La vaccination des personnes vulnérables est le moyen le plus efficace de prévenir la transmission de la maladie et les décès provoqués par la grippe. Un vaccin sécuritaire et efficace constitue la pierre angulaire de la préparation et de l'intervention en cas de pandémie. La Stratégie canadienne de vaccination en cas de pandémie repose sur l'efficacité des programmes de lutte contre la grippe saisonnière. Cependant, il y a des spécificités à prendre en compte lors d'une pandémie.

\section{Cadre de priorisation des vaccins pandémiques}

Il est peu probable que l'approvisionnement initial en vaccins contre la pandémie puisse être immédiatement suffisant pour répondre aux besoins de tous les Canadiens. Pour remédier à cette situation de manière juste, le Comité consultatif national de l'immunisation (CCNI) formulera des recommandations nationales relatives à l'utilisation prioritaire des vaccins en se servant du Cadre de priorisation des vaccins pandémiques jusqu'à ce qu'un nombre suffisant de vaccins soit à disposition pour l'ensemble des Canadiens (environ un mois après la mise à disposition des premiers lots) (4).

Le CCNI est un organisme consultatif auprès de l'Agence. II lui incombe de formuler des recommandations sur la base de conseils d'experts concernant l'ensemble des vaccins utilisés au Canada, dont les vaccins contre la grippe pandémique et la grippe saisonnière, par l'intermédiaire de son Groupe de travail sur l'influenza du CCNI (5). L'orientation offerte par le $\mathrm{CCNI}$ sur I'utilisation du vaccin en cas de pandémie sera mise à jour au besoin. Par exemple, les recommandations initiales peuvent évoluer à mesure que l'épidémiologie particulière d'une pandémie devient plus évidente.

Au début d'une pandémie, le cadre de priorisation offre une approche systématique pour prendre en compte l'ensemble des preuves scientifiques pertinentes tout en envisageant les considérations éthiques, logistiques et autres. Dans le cadre de ces recommandations, les groupes de risque et d'âge seront répartis dans divers groupes prioritaires au moyen des stratégies les plus efficaces pour atteindre les objectifs de lutte contre la pandémie à l'échelle nationale. Le cadre a été utilisé avec succès pour élaborer des lignes directrices de séquençage à l'occasion du déploiement du vaccin au cours de la pandémie de grippe pH1N1. Les groupes prioritaires définis étaient les suivants : les personnes de moins de 65 ans souffrant d'un état chronique; les femmes enceintes; les enfants âgés de 6 mois à 5 ans; les personnes se trouvant dans des milieux éloignés et isolés; les travailleurs de la santé prenant part à l'intervention contre la pandémie ou fournissant des services essentiels; les contacts familiaux et les fournisseurs de soins de santé à des personnes à risque élevé ne pouvant pas être vaccinées; et d'autres populations à risque élevé (6).

\section{Approvisionnement en vaccins}

La production nationale du vaccin contre la grippe est indispensable pour garantir l'accès du Canada à des quantités appropriées de vaccins. La raison tient au fait que l'obtention de vaccins produits à l'étranger peut être limitée au cours d'une pandémie en raison d'embargos sur les voyages ou sur les échanges commerciaux, ainsi que d'une demande accrue. Le Canada a été l'un des premiers pays à mettre en œuvre un contrat à long terme en matière d'approvisionnement en vaccins avec un fabricant de vaccins en 2001. La première occasion de profiter de ce contrat s'est présentée en 2009 lors de la pandémie de grippe pH1N1. Le bilan a montré qu'il y avait suffisamment de vaccins pour tous les Canadiens qui souhaitaient être vaccinés. Depuis lors, le Canada a conclu un autre contrat sur dix ans (qui a commencé en 2011). Le contrat stipule que le fabricant doit remplir ses engagements envers le Canada avant de vendre le vaccin à d'autres pays, ce qui garantit un approvisionnement adéquat pour chaque personne résidant au Canada. Dans le but d'assurer la capacité de fabrication du vaccin, le contrat actuel prévoit l'accès à une part du marché canadien des vaccins antigrippaux saisonniers.

\section{Réglementation sur les vaccins}

Tous les vaccins destinés à être administrés au Canada sont assujettis aux dispositions de la Loi sur les aliments et drogues (7) et du Règlement sur les aliments et drogues (8). Avant d'autoriser un nouveau vaccin, les experts de Santé Canada évaluent les données cliniques et scientifiques soumises par le fabricant. Le processus observé pour autoriser chaque année l'utilisation des vaccins antigrippaux saisonniers approuvés diffère du processus complet auquel sont soumis les nouveaux vaccins, puisque le changement ne touche que les souches de grippe utilisées. Des mois avant que la saison de la grippe commence, les fabricants envoient des renseignements mis à jour aux organismes de réglementation à propos des souches prévues pour la saison 
à venir. Santé Canada réalise alors un examen des données et autorise le nouveau vaccin saisonnier.

Les processus réglementaires normalisés ne peuvent pas être utilisés en cas de pandémie puisque la production d'un vaccin contre la grippe pandémique ne peut pas avoir lieu avant que la souche du virus n'ait été identifiée. Afin qu'un vaccin soit utile pour endiguer les effets de la pandémie, il doit être disponible presque immédiatement après sa fabrication. Que peut-on faire pour réussir l'examen réglementaire lorsque l'on dispose de peu de temps et de peu de données?

L'approbation réglementaire d'un vaccin contre la grippe pandémique peut être effectuée de trois façons. Premièrement, de manière similaire à un vaccin saisonnier, un prototype de vaccin contre la grippe pandémique peut disposer d'une autorisation de commercialisation préalable en fonction des données d'immunogénicité substitutives, puisque cela serait suivi d'une autorisation ultérieure de changement de souche. Deuxièmement, on peut utiliser la réglementation sur les drogues nouvelles pour usage exceptionnel mise en place en 2011 après la pandémie de grippe pH1N1 (9). Ces réglementations permettent à Santé Canada d'accélérer I'autorisation d'un vaccin contre la grippe pandémique en fonction des données portant sur les animaux accompagnées des données disponibles portant sur les humains, tant qu'une trousse qualité exhaustive est disponible et qu'un plan de surveillance rigoureux est en place après la commercialisation du vaccin. Enfin, si la réglementation sur les drogues nouvelles pour usage exceptionnel ne s'applique pas (par exemple, parce que la trousse qualité n'est pas complète), une ordonnance provisoire peut être émise par le ministère de la Santé fédéral, en fonction de l'état de l'urgence pour la santé publique. Une telle ordonnance provisoire peut suspendre certaines exigences de la Loi sur les aliments et drogues et du Règlement sur les aliments et drogues, ce qui permet la délivrance d'une autorisation limitée dans le temps, avec d'autres obligations liées aux promoteurs, comme la présentation en temps opportun des données cliniques et sur la qualité manquantes, ainsi que l'information suivant la commercialisation.

\section{Innocuité du vaccin}

En raison de l'envergure de la campagne de vaccination en cas de pandémie et des voies réglementaires liées aux drogues nouvelles pour usage exceptionnel, il faut porter une attention particulière à l'innocuité du vaccin afin de réduire tout risque et d'optimiser les bienfaits du vaccin. L'innocuité du vaccin contre la grippe pandémique est fondée sur l'infrastructure et sur les systèmes déjà en place pour assurer la surveillance des vaccins administrés systématiquement. La surveillance après commercialisation des effets secondaires suivant l'immunisation (ESSI) est réalisée par l'Agence de la santé publique du Canada et Santé Canada avec des partenaires provinciaux et territoriaux et d'autres intervenants clés. Malgré le fait que I'application réglementaire préalable à la commercialisation fournisse une quantité importante d'information, la surveillance après commercialisation est essentielle pour recueillir les déclarations d'effets secondaires suivant l'immunisation graves ou inattendus. Cette information est ensuite utilisée pour évaluer les vaccins pertinents et prendre des dispositions réglementaires, s'il y a lieu. Cependant, un vaccin distribué en vertu de la réglementation liée aux drogues nouvelles pour usage exceptionnel disposerait de peu de données après commercialisation, voire aucune, et les activités de surveillance existantes entreprises par l'Agence de la santé publique du Canada, Santé Canada et les partenaires provinciaux et territoriaux nécessiteraient une mise à l'échelle considérable.

Au cours d'une pandémie, il est essentiel que les renseignements sur les effets secondaires suivant l'immunisation soient transmis rapidement au portefeuille fédéral de la Santé (p. ex. Santé Canada et l'ASPC), qui regroupera les rapports et les analysera. Parmi les signaux d'innocuité des vaccins qui pourraient figurer dans les rapports sur les effets secondaires suivant l'immunisation, mentionnons l'augmentation de la fréquence ou de la gravité des événements connus pour être attribuables au vaccin antigrippal (p. ex. les réactions allergiques) ou des événements indésirables dont les liens étaient auparavant inconnus (p. ex. la narcolepsie). Les signaux doivent être rapidement étudiés pour permettre de déterminer les causes et, s'il y a lieu, de prendre les mesures nécessaires. Les mesures possibles comprennent des mises à jour de la monographie du produit, des modifications apportées aux recommandations sur la vaccination, des changements apportés aux méthodes d'administration et à la mise en quarantaine, ou un rappel d'un lot de vaccins par Santé Canada. Les principaux besoins en matière de surveillance améliorée de l'innocuité des vaccins et d'évaluation des déclarations de cas sont notamment des protocoles permettant de mener rapidement des enquêtes sur le terrain, des ressources analytiques et une connaissance des taux de référence des effets indésirables potentiels afin de les comparer à ceux qui sont observés ou prévus.

\section{Attribution, distribution et entreposage des vaccins}

Un plan d'attribution (à savoir la quantité de vaccins que chaque province et territoire va recevoir) sera mis au point par le Groupe de travail sur l'approvisionnement en vaccins, un forum fédéral-provincial-territorial visant à coordonner l'acquisition de vaccins et les programmes de vaccination. De manière similaire à la pandémie de grippe $\mathrm{pH} 1 \mathrm{~N} 1$, la distribution dans les provinces et territoires sera fonction du nombre d'habitants au moment du début de la pandémie, en commençant par les premiers lots disponibles et en poursuivant selon le cycle de production des vaccins.

Comme c'est le cas pour tous les vaccins, des lignes directrices doivent être respectées concernant l'entreposage et la manipulation adéquats. Par exemple, la chaîne du froid doit être respectée tout au long du processus de distribution. Des renseignements détaillés sont fournis dans les Lignes directrices nationales sur l'entreposage et la manipulation des vaccins pour les vaccinateurs (10).

\section{Administration du vaccin}

Afin d'assurer la distribution rapide du vaccin en cas de pandémie, il est important d'avoir recours à l'utilisation la plus efficiente des fournisseurs de soins de santé pour l'administration du vaccin et de disposer d'une infrastructure commune pour recueillir des données de surveillance. La plupart des provinces et territoires disposent d'un certain type de modèle de prestation hybride (dans lequel les pharmaciens, les infirmières en santé publique et les médecins administrent le vaccin) pour 
la grippe saisonnière. De plus, chaque province et territoire tient à jour son propre système d'information, en utilisant des bases de données électroniques ou des systèmes papier, pour suivre la couverture vaccinale. Cette incompatibilité entre les systèmes a rendu complexe le suivi du vaccin antigrippal saisonnier, et elle peut donner lieu à l'omission de renseignements. Au niveau national, l'Agence surveille l'administration du vaccin antigrippal saisonnier par le biais de l'Enquête sur la couverture vaccinale nationale contre la grippe saisonnière et l'Enquête sur la santé dans les collectivités canadiennes, mais cela présente une période de latence importante et ne peut pas servir en cas de pandémie. Dans le cas d'une pandémie, des systèmes d'information interexploitables qui permettent à plusieurs fournisseurs d'accéder aux dossiers de vaccination et de les saisir sont requis afin de fournir des estimations précises et en temps opportun de la couverture vaccinale. Des travaux sont en cours afin de mettre au point des registres provinciaux et territoriaux pour la surveillance de la grippe saisonnière et de la grippe pandémique.

\section{Discussion}

La Stratégie canadienne de vaccination en cas de pandémie repose sur l'efficacité des programmes de lutte contre la grippe saisonnière, et elle s'appuie sur les leçons tirées pendant la pandémie de grippe $\mathrm{pH} 1 \mathrm{~N} 1$. Depuis la pandémie de grippe $\mathrm{pH} 1 \mathrm{~N} 1$, la stratégie canadienne de vaccination en cas de pandémie a été améliorée par le biais d'un nouveau contrat de fabrication avec un fabricant canadien d'un vaccin en cas de pandémie; de nouveaux processus réglementaires permettent d'accélérer les approbations réglementaires; une surveillance améliorée de l'innocuité des vaccins; des efforts déployés en continu pour mettre au point les registres de vaccination.

La stratégie canadienne de vaccination est toujours sujette à des modifications lorsqu'il y a de nouveaux développements. Par exemple, les vaccins à base de végétaux comportent une nouvelle technologie qui pourrait être commercialisée pendant la durée de vie du contrat actuel du vaccin contre la grippe pandémique. Les planificateurs doivent envisager cette possibilité ainsi que les effets et les coûts potentiels à long terme de ces nouvelles technologies. D'autres développements en matière de recherche pourraient également indiquer qu'il faut passer en revue la stratégie.

\section{Conclusion}

La stratégie de vaccination contre la grippe pandémique constitue l'un des éléments de la stratégie de préparation en cas de pandémie du Canada. Cela représente un engagement continu au nom de l'ensemble des gouvernements fédéralprovinciaux-territoriaux pour une collaboration et pour veiller à ce que le Canada soit prêt en cas de grippe pandémique. Le Canada était bien préparé pour la pandémie de grippe $\mathrm{pH} 1 \mathrm{~N} 1$, et grâce aux leçons retenues suite à l'intervention, il est désormais encore mieux préparé à l'avenir.

\section{Conflit d'intérêt}

Aucun.

\section{Remerciements}

Membres du Groupe de travail sur la préparation du Canada en cas de grippe pandémique (GT PCPI) : B Henry (président), A Alfieri, S Gant, I Gemmil, T Hatchette, E Henry, B Schwartz, R Stirling

Secrétariat du GT PCPI : S Gadient, A House

ASPC : C Bell, L Cantin, G Charos, L Colas, R Pless, SE Smith, A Thom, K Watkins

Santé Canada : S Chung, F Lalonde, A Rinfret

\section{Financement}

Les travaux du Groupe de travail sur la préparation du Canada en cas de grippe pandémique sont financés par l'Agence de la santé publique du Canada.

\section{Références}

1. Agence de la santé publique du Canada. Leçons à retenir: Réponse de l'Agence de la santé publique du Canada et de Santé Canada à la pandémie de grippe H1N1 de 2009. Ottawa: Gouvernement du Canada; 2010. Disponible à I'adresse : http://www.phac-aspc.gc.ca/about_apropos/ evaluation/reports-rapports/2010-2011/h1n1/pdf/h1n1-fra. pdf.

2. Agence de la santé publique du Canada. Préparation du Canada en cas de grippe pandémique : Guide de planification pour les secteur de la santé [Internet]. Ottawa (ON): Gouvernement du Canada, 2015 [consulté le $1^{\text {er }}$ mai 2017]. Disponible à l'adresse : http://www.phac-aspc.gc.ca/ cpip-pclcpi/index-fra.php.

3. Agence de la santé publique du Canada. Préparation du Canada en cas de grippe pandémique : Guide de planification pour les secteur de la santé [Internet]. Ottawa: Gouvernement du Canada; 2017 [consulté le 1er mai 2017]. Annexe D, Préparation de l'intervention axée sur la vaccination en cas de pandémie. Disponible à l'adresse : http://www.phac-aspc.gc.ca/cpip-pclcpi/ann-d-fra.php.

4. Agence de la santé publique du Canada. Préparation du Canada en cas de grippe pandémique : Guide de planification pour les secteur de la santé [Internet]. Ottawa: Gouvernement du Canada; 2017 [consulté le 1 ${ }^{\text {er }}$ mai 2017]. Annexe, Cadre de priorisation des vaccins pandémique. Disponible à l'adresse : http// www.phac-aspc.gc.ca/cpippclcpi/ann-vf-fra.php.

5. Comité consultatif national de l'immunisation (CCNI) [Internet]. À propos du NACl. Ottawa: ASPC; 2017. Disponible à l'adresse : http://www.phac-aspc.gc.ca/naciccni/index-fra.php.

6. Agence de la santé publique du Canada. Guidance Document on the Use of Pandemic Influenza A (H1N1) 2009 Inactivated Monovalent Vaccine. October 21, 2009. Ottawa : ASPC. Disponible à l'adresse : http://centralhealth. nl.ca/assets/Pandemic-Influenza/Guidance-Document-on- 
the-Use-of-Pandemic-Influenza-A-H1N1-2009-InactivatedMonovalent-Vaccine.pdf.

7. Gouvernement du Canada. Loi sur les aliment et drogues (Justice). Ottawa: Site Web de la législation (Justice); 2017 [cconsulté le $1^{\text {er }}$ mai 2017]. Disponible à l'adresse : http:// laws-lois.justice.gc.ca/fra/lois/f-27/.

8. Gouvernement du Canada. Règlement sur les aliment et drogues (Justice). Ottawa : Site Web de la législation (Justice); 2017 [consulté le 1er mai 2017]. Disponible à l'adresse : http://laws-lois.justice.gc.ca/fra/reglements/ C.R.C.\%2C_ch._870/index.html.

9. Gouvernement du Canada. Ligne directrice - Exigences en matière de présentation et de renseignements relatives aux drogues nouvelles pour usage exceptionnel (DNUE)
[Internet]. Ottawa : Santé Canada; 2014 [consulté le 1er mai 2017]. Disponible à l'adresse : https://www.canada. $\mathrm{ca} / \mathrm{fr} /$ sante-canada/services/medicaments-produits-sante/ produits-biologiques-radiopharmaceutiques-therapiesgenetiques/information-demandes-presentations/lignesdirectrices/exigences-presentation-renseignements-droguesexceptionnel-dnue.html.

10. Gouvernement du Canada. Lignes directrices nationales sur l'entreposage et la manipulation des vaccins pour les vaccinateurs 2015 [Internet]. Ottawa : ASPC; 2015 [consulté le $1^{\text {er }}$ mai 2017]. Disponible à l'adresse : https://www.canada. $\mathrm{ca} / \mathrm{fr} /$ sante-publique/services/publications/vie-saine/lignesdirectrices-nationales-entreposage-et-manipulation-vaccinspour-vaccinateurs.html.

\section{Lignes directrices canadiennes sur les infections transmissibles sexuellement}

\section{Mise à jour de l'application mobile (mai 2017)}
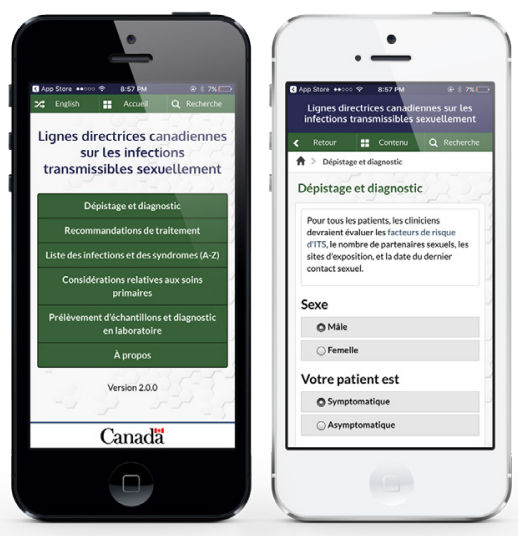

Nouveautés de la version 2.0.0

- Amélioration de l'interactivité avec des hyperliens internes

- Des boîtes contextuelles avec des renseignements supplémentaires et de conseils

- Des hyperliens externes vers des ressources complémentaires

Téléchargez-la aujourd'hui
Téléchargement gratuit

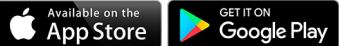

Recherchez: "Lignes directrices CDN ITS " 